\title{
Correlation Between Extended Radio and Line Emission for a Radio-Loud Quasar Sample
}

\author{
Xinwu Cao and D.R. Jiang \\ Shanghai Astronomical Observatory, Chinese Academy of Sciences, \\ Shanghai, National Astronomical Observatories, China
}

\begin{abstract}
A strong correlation is present between the extended radio and broad-line emission for a radio-loud quasar sample. The statistic behaviour on the core emission of lobe-dominated quasars is rather different from that of core-dominated quasars. The extended radio luminosity is a good tracer for jet power, while the core luminosity can only be a jet power tracer for core-dominated quasars.
\end{abstract}

\section{Introduction}

Relativistic jets have been observed in many radio-loud AGNs and are believed to be formed very close to the black holes. The jet-disc connection has been investigated by many workers (Rawlings \& Saunders 1991; Falcke \& Biermann 1995; Xu \& Livio 1999). An effective approach to study the link between these two phenomena is to explore the relationship between the corresponding emission. Rawlings \& Saunders (1991) presented a correlation between $Q_{\text {jet }}$ and the narrow line luminosity $L_{\mathrm{NLR}}$.

The broad-line region is ionized by the central source and is therefore a good indicator of emission from the accretion disc. VLA observations can separate the extended radio emission from the core emission. In this work, we use Cao \& Jiang's (1999) sample and collect all available data of extended radio emission from the literature to explore the relation between extended radio emission and broad-line emission.

\section{Results}

We find a strong correlation between $L_{\text {ext }}$ and $L_{\text {line }}$ at 99.9 per cent confidence (see Fig. 1). A correlation between $L_{\text {ext }}$ and $L_{\text {line }}$ (at 98 per cent confidence) exists for sources with $0.5<z<1.5$, while no correlation between $L_{\text {ext }}$ and $z$ is found for this subsample. There are significant correlations between fluxes. The Spearman partial rank correlation analysis has been performed for this sample, which confirms this conclusion. 


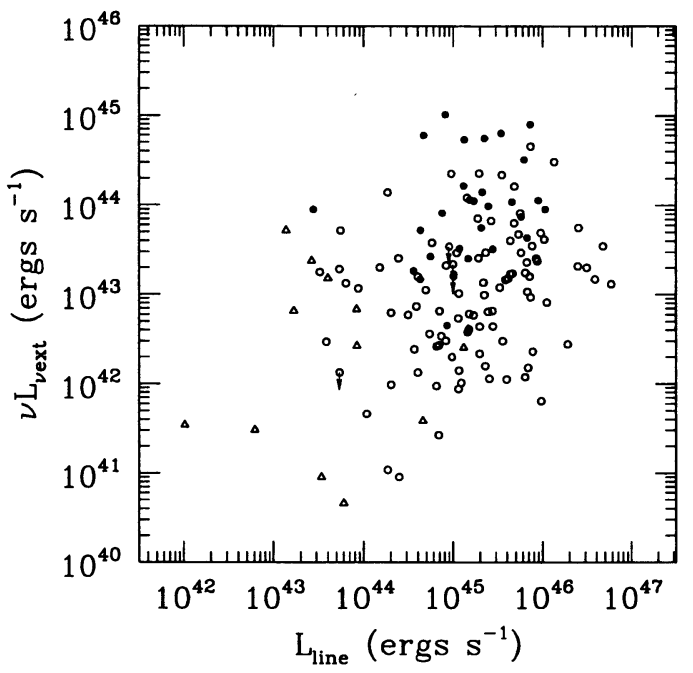

Figure 1. The extended radio and broad-line luminosity relation. The open circles represent core-dominated quasars(CDQs), and the full circles represent lobe-dominated quasars(LDQs), while the triangles represent BL Lac objects.

\section{Conclusions}

The core emission in flat-spectrum quasars is strongly beamed towards us, but the extended emission is not. The extended radio emission from flat-spectrum quasars can be taken as a tracer of jet power, as that for steep-spectrum quasars.

Most sources in our sample are CDQs (99 of 128, if we define CDQs as $R>1$ ). A significant intrinsic correlation is found between the extended radio luminosity and total broad-line luminosity (the Spearman partial rank correlation analyses confirm this result), which indicates a physical link between jets and accretion processes.

\section{References}

Cao X., Jiang D.R., 1999, MNRAS 307, 802

Falcke H., Biermann P., 1995, A\&A 293, 665

Rawlings S. G., Saunders R. D. E., 1991, Nat 349, 138

Xu C., Livio M., 1999, AJ 118, 1169 